\title{
A STUDY ON LINES OF FRICTION AND GRAVITATION THESIS
}

\author{
Mr. Soham Mohan Dalal \\ St. Teresa con. Higher School, Jalgaon \\ Jalgaon, Dist. Jalgaon, (Maharashtra state), INDIA
}

\begin{abstract}
- what co-relates between the line of friction and line of gravitation?, what is the value of a line of friction when the body performs inverse motion?, what is the value of shortest path i.e. displacement between the distance of the line of friction and the distance of the line of gravitation? Do you know the measurement of the angle formed due to the line of gravity and line of friction? All such question is letting the mind to think on the topic that what is it? , Do the infinite rays of drag force can be the height of an object? All these questions would clarify accordingly when you start reading the entire article. The article is going to deal with the questions raised above and will introduce you to the concepts and laws between the line of friction and gravity when the body performs a motion. Here there are two types of motion - 1) Inverse (reverse) motion 2) motion when the body is away from the ground surfaces. The article is also going to introduce the two laws of 1 l.s (friction and gravitation) Thesis
\end{abstract}

Aim to study: The main aim of this article is to study the co-relation between 11.s (friction and gravitation) and the laws depend on this concept. The new concept of finding the height of the object when the body is away from the earth's surface concerning the concept of skin friction. How this skin friction can affect the body when falling on the ground and the infinite rays of drag force directly proportional to the ll.of gravitation can relate to find the height of object and what is this 11.s of gravitation and friction and how they can result in finding the displacement between them.

Keywords: Ramanujan Summation of sums of all-natural numbers till infinite, trigonometric functions, ratios, and applications. Using the approx. values for the radius of earth $=$ $6.4 \times 10^{\wedge} 6 \mathrm{~m}$ and universal gravitational constant $=6.67 \times 10^{\wedge}$ $11 \mathrm{Nm}^{\wedge} 2 / \mathrm{kg}^{\wedge} 2, \mathrm{r}=$ radius of the earth, $\mathrm{d}=$ displacement between distances of 11.s of friction and gravitation, 1l. (f) =line of friction, $n=$ the infinite numbers in counting, $h=$ height of the object

\section{INTRODUCTION}

Now let us introduce the common basic concepts regarding this article using diagrams,

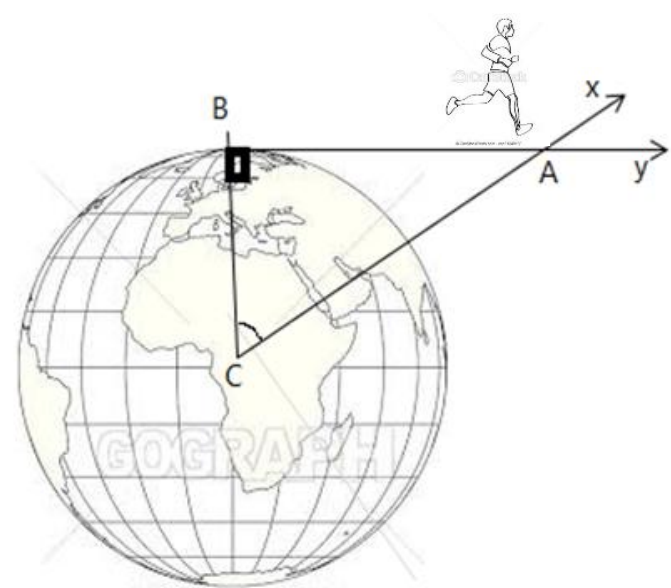

Fig..1

Now here in fig. 1 ,

$\mathrm{A}$ is the object at the initial position at $\mathrm{A}$ and its final position is at B after performing an Inverse motion. Now ray BY is a 11 . of friction because frictional force always acts in the opposite Direction of motion of an object so here motion was in the direction to the final point $\mathrm{B}$ and so friction 1l. is in opposite to Final point.

11. $\mathrm{AC}=6.4 \times 10^{\wedge} 6$ is the radius of the earth with Centre $\mathrm{C}$. AC is a line of gravitation which is pulling an object towards earth due to the force of gravity. Ray CX is a ll. of displacement Between distances of 1l.s of friction and gravitation which intersects at points $\mathrm{A}$ on 11. of friction. Angle $\mathrm{ABC}=90^{\circ}$ because of 11. BC perpendicular on ray BY.

1.1 The thesis of 1st law of displacement and ll.gravity

1st Law proves that II. $(d)=11$. $(r)$ - 


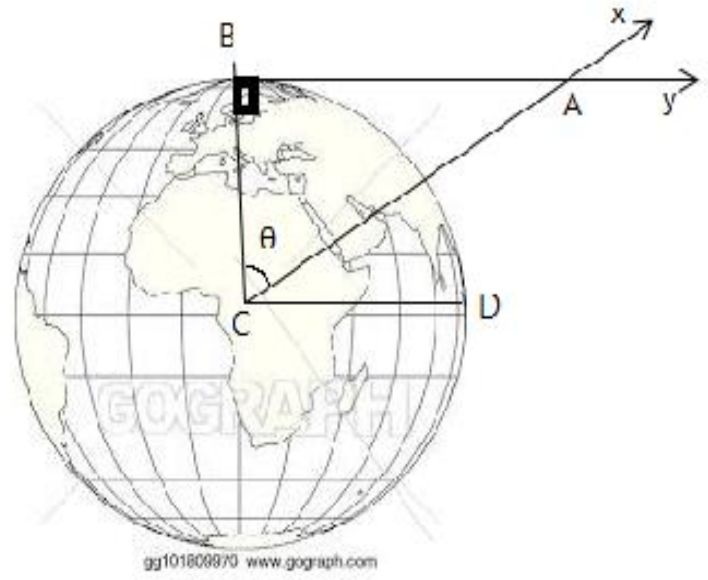

Fig. 2

Given: In fig.2, angle of elevation $(\theta)$ i.e. angle $B A X=60^{\circ}$ and $\mathrm{CD}=\mathrm{BC} \ldots$ (same radii of circle), $\mathrm{r}=$ radius of earth, $\mathrm{d}=$ displacement between distances of ll.s of friction and gravitation.

Methodology: line AB // line CD

Angle $\mathrm{BCA} \cong$ angle $\mathrm{BAX} \ldots$.. (Corresponding angles)

I.e. angle $\mathrm{BCA}=60^{\circ}$

From figure, $\tan \theta=\frac{A B}{B C}$

$\therefore \tan \theta=60^{\circ}$

$\therefore \sqrt{3}=\frac{u(f)}{r}$

$\sqrt{3} \times \mathrm{r}=11 .(\mathrm{f}) \ldots$ (3)

NOW, $\therefore \operatorname{Sin} \theta=\frac{A B}{A C}$

$$
\begin{gathered}
\therefore \operatorname{Sin} \theta=60^{\circ} \ldots(5) \\
\sqrt{3}=\frac{u_{(f)}}{d} \ldots(4 \text { and } 5) \\
\frac{\sqrt{a}}{1}=\frac{\sqrt{\mathrm{ax} x}}{d} . .(\text { From } 3)
\end{gathered}
$$

$$
\sqrt{3} \times d=\sqrt{3} \times r
$$

$\mathrm{d}=\mathrm{r}$

$\therefore$ Radius of earth $=$ displacement between distances of 11 . of friction and gravitation

$\therefore$ Hence proved $1^{\text {st }}$ law of displacement and ll.gravity

\subsection{The thesis of ll.Drag force and ll.gravity}

$2^{\text {nd }}$ law proves that $\frac{[\operatorname{mn}+n[d]]}{G}=h$

By using the concept of skin friction which is when an object falls on the ground then, the resistance force i.e. drag force caused the motion of the body when traveling through the medium of fluid or air. Always the drag force acts opposite to the direction of approaching the flow velocity of an object.

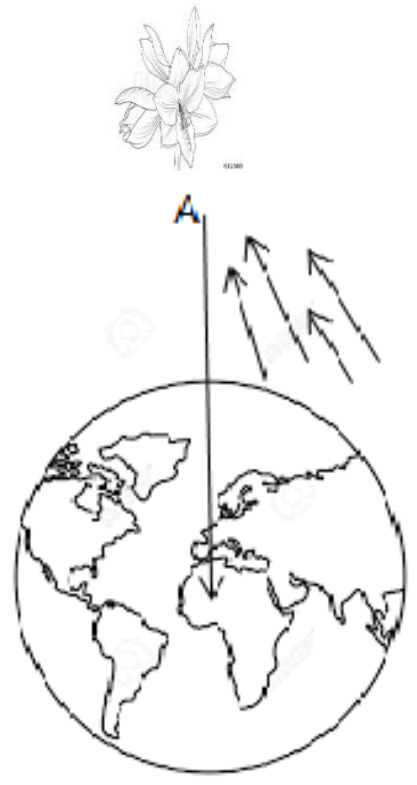

Fig.3

In fig.3,

Now here at point A some flowers are falling on the ground so now at the initial point when flowers are away from earth surface at point A they receive a force of gravity from the Centre of earth.

The motion of flowers while falling on the ground caught in resistance force i.e. drag force because it is traveling through the medium of air. It is called skin friction because here flowers a solid layer comes in contact with air molecules and approaching the drag force to the flowers and these forces ll.s are in infinite in counting. As shown in figure-3

$\mathrm{n}$ refers to the infinite rays of drag force exerting on flowers at point $\mathrm{A}$ and $\mathrm{X}$ is a single ray of drag force Now 1l.s of drag force directly proportion to ll.gravity. To show calculations between two forces we use universal gravitational constant $=$ $\mathrm{G}$ according to the newton law of universal law of gravitation. According to the direct proportion,

$\therefore \mathrm{N}(\mathrm{X})=\mathrm{h} \times \mathrm{G} \ldots .(7)$

Now rays are till infinite so, $\mathrm{t} 1=$ ray $1, \mathrm{t} 2=$ ray $2, \mathrm{t} 3=$ ray $3, \cdots$ $\cdots, \mathrm{tn}=$ rayn

$1,2,3,4 \ldots . . \mathrm{n}$ 
$t 2-t 1=d, 2-1=1$

$\therefore t 3-t 2=d$

$\therefore 3-2=1$

Here 1 is common difference $=\mathrm{d}$ and sequence is of arithmetic progression.

The sequence in arithmetic progression is a, $(a+d)$, $(a+2 d),(a+3 d), \ldots \ldots \ldots \ldots \ldots . . . .(a+n d)$

I.e. infinite numbers in A.P,

$=[\operatorname{tn}+\mathrm{n}(\mathrm{d})]=\mathrm{n}(\mathrm{x}) \ldots .(8)$

Now putting $n(x)=[\operatorname{tn}+n(d)]$ in eq( 7),

$\frac{[\operatorname{tn}+n[d]}{G}=h$

$\frac{\mathrm{t} 1+\mathrm{n}(1)}{G}=h$

$\frac{t 1+n}{G}=h$

Now here $t 1+n=-1 / 12$

According to the Srinivasa Ramanujan, et al. (1918) in their work he states that if adding all natural numbers, i.e. 1, 2, 3, 4 , and so on, all the way to infinity, sum is equal to $-1 / 12$.)

$\therefore$ Hence proved $2^{\text {nd }}$ law of 11.Drag force and 1l.gravity

\section{RESULT}

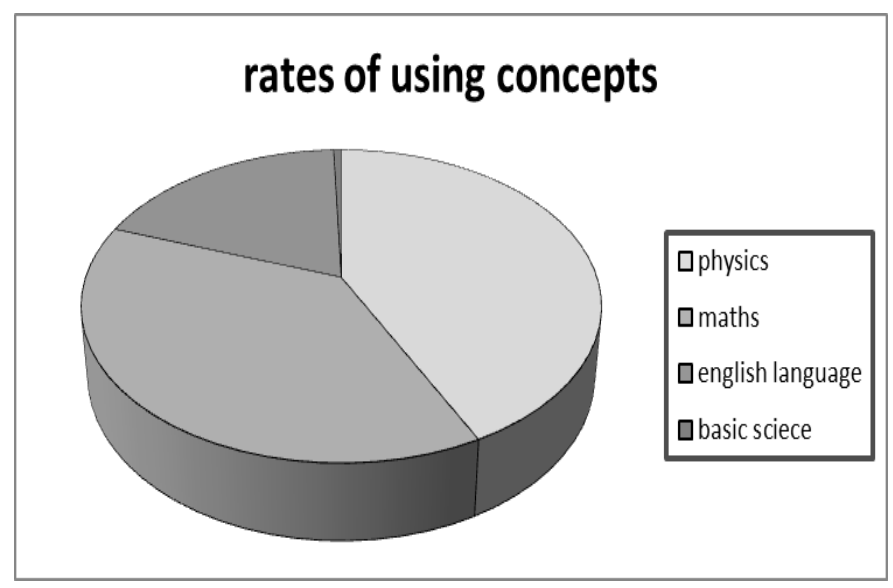

Author's information - St. teresa con.high school, Jalgaon, Dist- Jalgaon, Maharashtra, India.

\section{List of abbreviations used -}

\begin{tabular}{|l|l|}
\hline Full forms & Abbreviated form \\
\hline Line & 11. \\
\hline Lines & $11 . s$ \\
\hline Arithmetic progression & A.P. \\
\hline
\end{tabular}

\section{CONCLUSION}

By these laws, the world may introduce to the new way of the concept of 11.s (friction and gravitation).this law represent the truth and also gave the world the formula for finding the height of an object with relation to infinite rays of drag force and stated that radius of earth $=$ displacement between distances of 11. of friction and gravitation. The complete proof you had got using some notions of math and physics in these laws of ll.s (friction and gravitation).

Acknowledgment: This work supported by the author itself. It is self-research any another second correspondence is not involved. Country Name-India.

\section{REFERENCES}

[1] Ramanujan S.,(1918) sums in a 1918 paper Rumanujan's sums are used in the proof of Vinogradov's theorem that every sufficiently-large odd number is the sum of three primes.(in hardy ramanujan journal).

[2]Ramanujan, Srinivasa (1916), "On Certain Arithmetical Functions", Transactions of the Cambridge Philosophical Society, 22 (9): 159-184 (pp. 136-163 of his Collected Papers)

[3] Nicol, C. A. (1962). "Some formulas involving Ramanujan sums". Can. J. Math. 14: 284-286. doi:10.4153/CJM-1962019-8.

[4] Ramanujan, Srinivasa (2000), Collected Papers, Providence RI: AMS / Chelsea, ISBN 978-0-8218-2076-6

[5] Hardy, G. H.; Wright, E. M. (1979) [1938]. An Introduction to the Theory of Numbers (5th ed.). Oxford: Clarendon

Press. ISBN 0-19-853171-

0. MR 0568909. Zbl 0423.10001.

[6] Hazewinkel, Michiel, ed. (2001) [1994], "Arithmetic series", Encyclopedia of Mathematics, Springer Science+Business Media B.V. / Kluwer Academic Publishers, ISBN 978-1-55608-010-4Weisstein, Eric W. "Arithmetic p

[7] Weisstein, Eric W. "Arithmetic series". MathWorld.

[8] Sigler, Laurence E. (trans.) (2002). Fibonacci's Liber Abaci. Springer-Verlag. pp. 259-260. ISBN 0-387-95419-8.

[9] White, Frank (2011). Fluid Mechanics. New York City, NY: McGraw-Hill. pp. 477-478. ISBN 9780071311212.

[10]^ Nitsche, W.; Thünker, R.; Haberland, C. (1985). A computational Preston tube method. Turbulent shear flows, 4. pp. 261-276.

[11]^ Prandtl, L. (1925). "Bericht uber Untersuchungen zur ausgebildeten Turbulenz". Zeitschrift fur angew. Math. u. Mechanik 5.2: 136-139.

[12]^ White, Frank (2011). Fluid Mechanics. New York City, NY: McGraw-Hill. pp. 484-485. ISBN 9780071311212. 
[13]^ Incropera, Frank; Bergman, Theodore; Lavine, Adrienne (2013). Foundations of Heat Transfer. Hoboken, NJ: Wiley. pp. 402-404. ISBN 9780470646168.

[14]Chapman, G. T. Topological classification of flow separation on three-dimensional bodies AIAA, Aerospace Sciences Meeting, 24th, Reno, NV, Jan. 6-9, 1986. 22 p.[1]

[15] Isaac Newton: "In [experimental] philosophy particular propositions are inferred from the phenomena and afterwards rendered general by induction": "Principia", Book 3, General Scholium, at p.392 in Volume 2 of Andrew Motte's English translation published 1729.

[16] Purrington, Robert D. (2009). The First Professional Scientist: Robert Hooke and the Royal Society of London. Springer. p. 168. ISBN 978-3-0346-0036-1. Extract of page $\underline{168}$

[17]See J. Bruce Brackenridge, "The key to Newton's dynamics: the Kepler problem and the Principia", (University of California Press, 1995), especially at pages 20-21.

[18] Hazewinkel, Michiel, ed. (2001) [1994], "Trigonometric functions", Encyclopedia of Mathematics, Springer Science+Business Media B.V. / Kluwer Academic Publishers, ISBN 978-1-55608-010-4

[19] O'Connor, J. J., and E. F. Robertson, "Trigonometric functions", MacTutor History of Mathematics archive. (1996)

[21]Kantabutra,Vitit, "On hardware for computing exponential and trigonometric functions," IEEE Trans. Computers 45 (3), 328-339 (1996).

[22] Trope Y, Liberman N (April 2010). "Construal-level theory of psychological distance". Psychological Review.

[23]Tom Henderson. "Describing Motion with Words". The Physics Classroom. Retrieved 2 January 2012.

$[24]_{-}^{\wedge}$ Stewart, James (2001). "§2.8 - The Derivative As A Function". Calculus (2nd ed.). Brooks/Cole. ISBN 0-53437718-1. 